\title{
卤化物钙钛矿射线探测器材料研究进展
}

\author{
孟 钢 $^{1}$, 叶雨琪 ${ }^{1,2}$, 范黎明 ${ }^{1}$, 王时茂 ${ }^{1}$, GNATYUK Volodymyr ${ }^{3}$, 方晓东 ${ }^{1}$ \\ (1. 中国科学院安徽光学精密机械研究所, 合肥 $230031 ; 2$. 中国科学技术大学, 合肥 $230026 ; 3$. 乌克兰科学院 \\ 半导体物理研究所, 基辅 03028 , 乌克兰)
}

摘 要: 卤化物钻钣矿由于具有高射线吸收系数、高载流子迁移率寿命乘积、可低温溶液法生长等特性，有望突破 传统高纯锗和碲锌镉探测器在成本、芯片兼容性和大尺寸成像等方面的制约, 成为新一代性能优异的室温射线探测 材料。本文从卤化物钙钛矿材料的基本性质与射线探测原理出发, 介绍了 2015 年以来卤化物钻钛矿射线探测材料 与器件的发展历程; 系统介绍了直接型射线探测器(强度、成像、能谱)及闪炼体探测器的最新研究成果; 分析了材 料特性与器件结构对射线探测器性能的影响机制, 为今后更高效的卤化物销钛矿射线探测器的开发提供参考。

关 键 词: 卤化物钲铁矿; 射线探测器; 成像; 能谱; 闪炼体; 综述

中图分类号: O430 文献标识码: A

\section{Recent Progress of Halide Perovskite Radiation Detector Materials}

\author{
MENG Gang ${ }^{1}$, YE Yuqi ${ }^{1,2}$, FAN Liming ${ }^{1}$, WANG Shimao ${ }^{1}$, GNATYUK Volodymyr ${ }^{3}$, FANG Xiaodong ${ }^{1}$ \\ (1. Anhui Institute of Optics and Fine Mechanics, Hefei Institutes of Physical Science, Chinese Academy of Sciences, Hefei \\ 230031, China; 2. University of Science and Technology of China, Hefei 230026, China; 3. V.E Lashkaryov Institute of Semi- \\ conductor Physics of the National Academy of Sciences of Ukraine, Kyiv 03028, Ukraine)
}

\begin{abstract}
Owing to high $\mathrm{X} / \gamma$-ray absorption coefficient, high carrier mobility lifetime product, and low temperature solution growth, halide perovskites emerged as promising room temperature radiation detector materials, which outperform traditional high-purity Ge and CdZnTe materials in term of low-cost, chip compatibility and large-area imaging. Starting from the fundamental properties of halide perovskites and the principle of radiation detectors, the development of halide perovskite radiation detectors since 2015 was briefly introduced. Then, recent progresses of direct radiation detectors (intensity, imaging, energy spectroscopy) and indirect scintillator detectors were systematically reviewed, and the crucial factors for high-performance detectors were discussed, which could provide valuable guidance for further boosting performance of halide-perovskite-based radiation detectors in future.
\end{abstract}

Key words: halide perovskite; radiation detector; imaging; energy spectroscopy; scintillator; review

作为高能射线感知的元器件, 射线探测器广泛 应用于材料/高能物理研究、医学影像、安防安检、
环境监测等新兴领域 ${ }^{[1-4]}$ 。卤化物钙钛矿具有高射线 吸收系数、高迁移率、长载流子寿命、合适的带隙、

收稿日期：2019-07-31; 收到修改稿日期：2019-08-22

基金项目：国家自然科学基金(11604339,11674324); 中国科学院“百人计划”; 核探测与核电子学国家重点实验室课题 (SKLPDE-KF-201907); 量子光学与光量子器件国家重点实验室开放课题(KF201901) National Natural Science Foundation of China (11604339, 11674324); CAS Pioneer Hundred Talents Program; State Key Laboratory of Nuclear Detection and Nuclear Electronics (SKLPDE-KF-201907); National Key Laboratory of Quantum Optics and Photonic Devices (KF201901)

作者简介: 孟 钢(1982-), 教授. E-mail: menggang@aiofm.ac.cn MENG Gang(1982-), professor. E-mail: menggang@aiofm.ac.cn

通讯作者: 方晓东, 教授. E-mail: xdfang@aiofm.ac.cn FANG Xiaodong, professor. E-mail: xdfang@aiofm.ac.cn 
及独特的缺陷容忍与可低温溶液法生长等特性, 有 望克服现有高纯锗 $(\mathrm{HPGe})$ 和碲锌镉 $(\mathrm{CdZnTe}, \mathrm{CZT})$ 探测器在成本及灵敏度等方面的不足, 推动可室温 工作、低成本、高清、高灵敏(低剂量成像)、高能 谱分辨射线探测器的开发, 迅速成为当今研究热点 之一。

\section{1 卤化物钙钛矿材料特性及在射线探 测器中的优势}

\section{1 卤化物钙钛矿材料结构}

理想的卤化物钙钛矿材料 $\left(\mathrm{ABX}_{3}\right)$ 为面心立方 结构 ${ }^{[5]}$, 如图 1(a)所示。顶点 $\mathrm{A}$ 位为金属或有机离 子 $\mathrm{Rb}^{+} 、 \mathrm{Cs}^{+} 、 \mathrm{CH}_{3} \mathrm{NH}_{3}{ }^{+}\left(\mathrm{MA}^{+}\right)$和 $\mathrm{CH}\left(\mathrm{NH}_{2}\right)_{2}^{+}\left(\mathrm{FA}^{+}\right)$等; 体心 $\mathrm{B}$ 位为 +2 价的 $\mathrm{Sn}^{2+} 、 \mathrm{~Pb}^{2+}$ 等; 面心 $\mathrm{X}$ 位为卤素 离子 $\mathrm{Cl}^{-} 、 \mathrm{Br}^{-} 、 \mathrm{I}^{-[6]}$ 。其三维结构的稳定性取决于容 差因子 $t(0.89<t<1)$ :

$$
t=\frac{R_{\mathrm{A}}+R_{\mathrm{X}}}{\sqrt{2}\left(R_{\mathrm{B}}+R_{\mathrm{X}}\right)}
$$

$R_{\mathrm{A}} 、 R_{\mathrm{B}} 、 R_{\mathrm{X}}$ 分别为 $\mathrm{A} 、 \mathrm{~B} 、 \mathrm{X}$ 位离子半径 ${ }^{[7-8]}$ 。由于 $\mathrm{B}$ 位通常是离子半径较大的 $\mathrm{Sn}^{2+} 、 \mathrm{~Pb}^{2+}$ 等离子, 所以 $\mathrm{A}$ 位离子半径较大时有利于立方结构的稳定。例如, $\mathrm{FA}^{+}(0.253 \mathrm{~nm})$ 取代 $\mathrm{MA}^{+}(0.217 \mathrm{~nm})$ 时结构会更加稳 定 ${ }^{[9-12]}$ 。双钙铁矿材料 $\left(\mathrm{A}_{2} \mathrm{~B}^{+} \mathrm{B}^{3+} \mathrm{X}_{6}\right)$ 是近年来涌现的 一类新材料, $\mathrm{B}$ 位包含一种 +1 价离子 $\left(\mathrm{Li}^{+} 、 \mathrm{Na}^{+}\right.$、 $\left.\mathrm{K}^{+} 、 \mathrm{Rb}^{+} 、 \mathrm{Ag}^{+}\right)$和一种 +3 价离子 $\left(\mathrm{Al}^{3+} 、 \mathrm{Ga}^{3+} 、 \mathrm{In}^{3+} 、\right.$ $\mathrm{Tl}^{3+} 、 \mathrm{Bi}^{3+} 、 \mathrm{Sb}^{3+}$ 等), 如图 1(b)所示。其结构的稳定 性需同时满足 $0.81<t<1.11$ 和 $0.44<\mu<0.90$ ( $\mu$ 为八面 体因子):

$$
t=\frac{R_{\mathrm{A}}+R_{\mathrm{X}}}{\sqrt{2}\left(\frac{R_{\mathrm{B}^{+}}+R_{\mathrm{B}^{3+}}}{2}+R_{\mathrm{X}}\right)}
$$

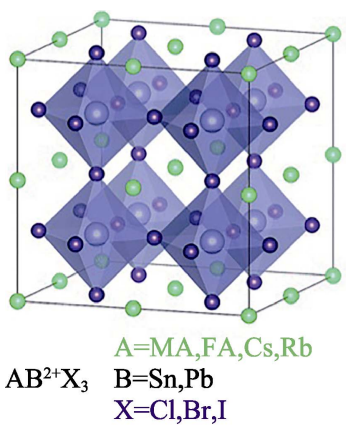

(a) Single perovskite

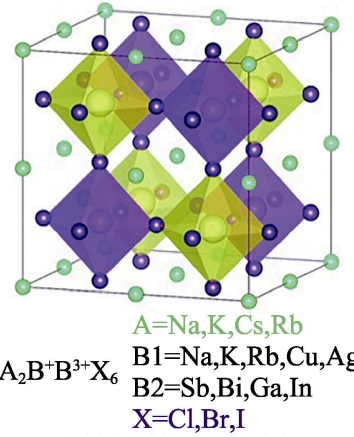

(b) Double perovskite

图 1 单钙钣矿 (a) 与双销铁矿 (b) 的晶体结构示意图 ${ }^{[5]}$

Fig. 1 Crystal structures of (a) single $\left(\mathrm{AB}^{2+} \mathrm{X}_{3}\right)$ and (b) double $\left(\mathrm{A}_{2} \mathrm{~B}^{+} \mathrm{B}^{3+} \mathrm{X}_{6}\right)$ halide perovskite ${ }^{[5]}$

$$
\mu=\frac{R_{\mathrm{B}^{+}}+R_{\mathrm{B}^{3+}}}{2 R_{\mathrm{X}}}
$$

其中, $R_{\mathrm{B}^{+}} 、 R_{\mathrm{B}^{3+}} 、 R_{\mathrm{X}} 、 R_{\mathrm{A}}$ 分别为 $\mathrm{B}^{+} 、 \mathrm{~B}^{3+} 、 \mathrm{X} 、$ $\mathrm{A}$ 位离子半径 ${ }^{[13]}$ 。卤化物钙钛矿材料的光电性能主 要由 $\left[\mathrm{BX}_{6}\right]$ 八面体决定, 双钙钛矿材料 $\mathrm{B}$ 位原子的 多样性为其光电性能的调控提供了充足的自由度。

\section{2 卤化物钙钛矿材料在射线探测中的优势}

卤化物钙钛矿材料性能优异: 通过调节 A 位、 $\mathrm{X}$ 位离子种类及比例可实现带隙在可见-紫外波段 $(1.6 \sim 3.0 \mathrm{eV})^{[14-16]}$ 及光致发光谱(PL)峰位(图 2(a)) 的准连续调节 ${ }^{[17]}$, 可见光波段光子吸收系数高 $\left(\sim 10^{5} \mathrm{~cm}^{-1}\right)^{[18]}$ 、载流子迁移率高 ${ }^{[19-21]}$ 、缺陷密度

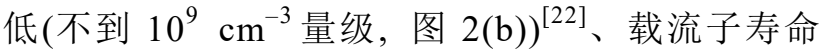

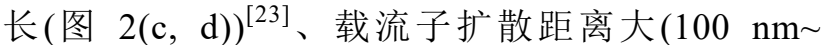

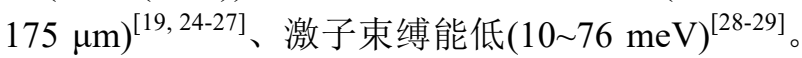
由于卤化物钙钛矿常含 $\mathrm{Cs} 、 \mathrm{~Pb} 、 \mathrm{Br} 、 \mathrm{I}$ 等高原子序数 (Z)原子, 可高效地吸收高能射线光子的能量(射线吸 收/衰减系数 $\alpha \propto \frac{Z^{4}}{E^{3}}, E$ 是射线光子能量)。吸收 $99 \%$ 以上 $(50 \mathrm{keV}) \mathrm{X}$ 射线所需钙钛矿 $\left(\mathrm{MAPbBr}_{3}\right.$ 或 $\mathrm{Cs}_{2} \mathrm{AgBiBr}_{6}$ 的厚度分别为 $2.28 \mathrm{~mm}$ 或 $1.18 \mathrm{~mm}^{[30]}$, 仅为 $\mathrm{Si}$ 厚度的 $1 / 10$ 左右, 如图 2(e, f) 所示。探测器 尺寸的大幅度降低不仅有利于降低光生电子一空穴 对的复合几率，同时也利于高清、超薄射线成像(相 机)的开发。

\section{3 射线探测器的类型及发展历程}

射线与卤化物钙钛矿材料相互作用过程如图 3 所示: 卤化物钲钛矿材料吸收高能射线光子能量 (1) 产生热空穴、热电子; 热化后(2)驰豫到材料价带 底、导带顶; (3)形成电子-空穴对; 这些非平衡的载 流子可通过辐射 (可见)光子的途径(间接型/闪炼 体探测器), 或以光电流 (直接型探测器)的途径检 测 (4)。值得一提的是，卤化物钻钛矿是现有射线探 测材料中唯一可用作直接型与间接型探测器的材 料。自 2015 年 Yakunin 等 ${ }^{[21]}$ 首次报道 $\mathrm{MAPbI}_{3}$ 多晶 厚膜 $(\sim \mu \mathrm{m})$ 探测器以来, 低剂量、高灵敏 $\mathrm{X} / \gamma$ 射线探

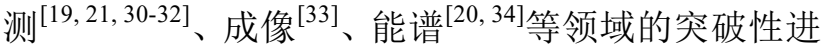
展不断涌现(图 4)。

\section{2 卤化物钲钛矿辐射探测器}

\section{1 直接型探测器}

\subsection{1 射线探测}

2015 年, Yakunin 等 ${ }^{[21]}$ 利用有机一无机杂化钙钛 矿材料 $\alpha$ 值较大的优势, 首次将钻钛矿光电探测器波 段从可见-紫外 ${ }^{\left[{ }^{[35-38]}\right.}$ 拓展到 $\mathrm{X}$ 射线波段。 $\mathrm{MAPbI}_{3}$ 多 
晶厚膜 $(0.2 \sim 2 \mu \mathrm{m})$ 探测器响应速度较快, 灵敏度达到 $25 \mu \mathrm{C} \cdot \mathrm{mGy}_{\mathrm{air}}{ }^{-1} \cdot \mathrm{cm}^{-3}$ (图 5(a)右纵轴), 优于当今临床平 板成像仪所用的 $\alpha-\mathrm{Se}$ 探测器 $\left(1 \sim 17 \mu \mathrm{C} \cdot \mathrm{mGy}_{\mathrm{air}}{ }^{-1} \cdot \mathrm{cm}^{-3}\right)$ 。 然而, 多晶膜(存在大量空隙、晶界及表面缺陷)的 的载流子迁移率 $(\mu)$ 与寿命 $(\tau)$ 的乘积 $(\mu \tau)$ 仅为 $2 \times$ $10^{-7} \mathrm{~cm}^{2} \cdot \mathrm{V}^{-1}$ (比 CZT 小 5 个数量级 ${ }^{[3,39]}$ ), 不利于光生 电子-空穴对的快速分离。2016 年, Wei 等 ${ }^{[19]}$ 首次 报道了单晶 $\mathrm{MAPbBr}_{3}(\sim 1 \sim 2 \mathrm{~mm}$ 厚 $) \mathrm{X}$ 射线探测器, 优化后的 $\mu \tau$ 达到 $1.2 \times 10^{-2} \mathrm{~cm}^{2} \cdot \mathrm{V}^{-1}$, 灵敏度达到 $80 \mu \mathrm{C} \cdot \mathrm{Gy}^{-1} \cdot \mathrm{cm}^{-2}$, 比相同偏压下 $\mathrm{CZT}$ 探测器高 10 倍
以上 ${ }^{[40]}$ 。探测器在接近零偏压的情况下可检测到的 最低 $X$ 射线剂量率为 $0.5 \mu \mathrm{Gy}_{\mathrm{air}} \cdot \mathrm{s}^{-1}$ (图 5(b)), 远低于 常规医疗诊断所需的剂量率 $\left(5.5 \mu \mathrm{Gy}_{\mathrm{air}} \cdot \mathrm{s}^{-1}\right)^{[41-42]}$ 。作 为一种新型室温辐射探测材料, 有机一无机杂化铅 基卤化物钙钛矿材料在过去四年发展迅速，然而材 料中含铅和有机阳离子带来不可忽视的毒性及稳定 性问题。有机一无机杂化钲钛矿材料的化学、热稳定

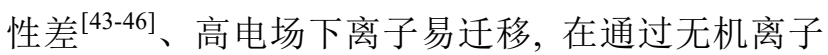
掺杂提升其结构及热稳定性的同时 ${ }^{[47-62]}$, 开发全无 机的无铅钙钛矿材料也得到了广泛的关注。
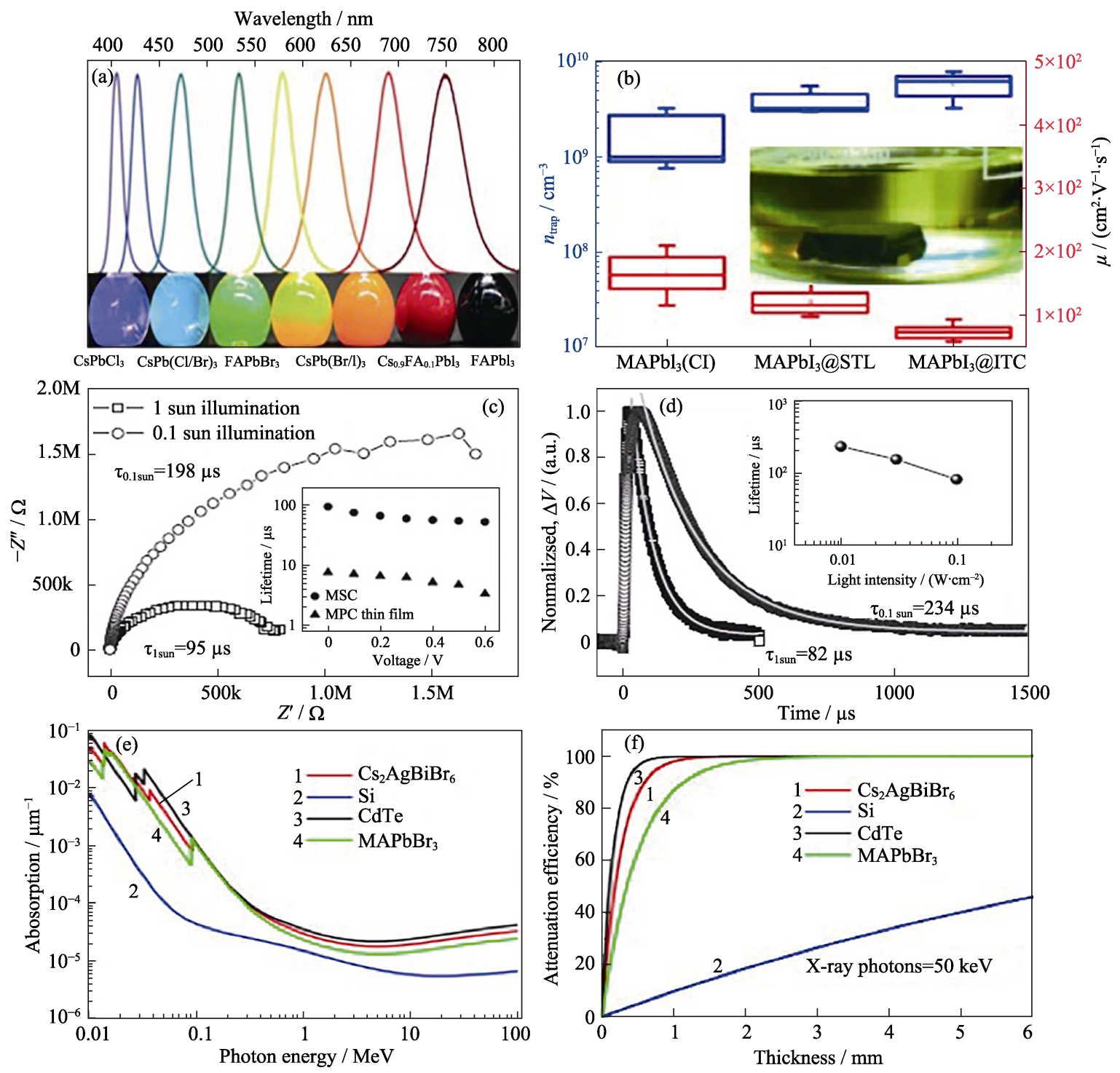

图 2 卤化物钲钛矿光电性能(a)X 位卤素离子及 $\mathrm{A}$ 位离子掺杂调节卤化物钻钛矿的光致发光峰 ${ }^{[17]}$; (b)溶液法生长的 $\mathrm{MAPbI}_{3}$ 单晶的缺陷浓度及载流子迁移率 ${ }^{[22]} ;(\mathrm{c}) \mathrm{MAPbI}_{3}$ 单晶的载流子寿命(电化学阻抗谱分析 ${ }^{[23]} ;(\mathrm{d}) \mathrm{MAPbI}_{3}$ 单晶的 载流子寿命(TPV 衰减曲线) $)^{[23]}$; (e)不同能量下的射线吸收系数 ${ }^{[30]}$; (f)不同厚度下的 $50 \mathrm{keV}$ 射线衰减效率 ${ }^{[30]}$

Fig. 2 Optoelectronic properties of halide perovskites. (a) Photoluminescence spectra of mixed halide perovskites ${ }^{[17]}$;

(b) Trap densities and carrier mobilities of $\mathrm{MAPbI}_{3}$ single crystals grown by solution method ${ }^{[22]}$; (c) Extracted carrier lifetime of $\mathrm{MAPbI}_{3}$ polycrystalline (MPC) film and single crystal (MSC) by EIS study ${ }^{[23]}$; (d) Extracted carrier lifetime of $\mathrm{MAPbI}_{3}$ single crystal (TPV decay curves) ${ }^{[23]}$; (e) Absorption coefficients versus photon energy of $\mathrm{X} / \gamma$-ray ${ }^{[30]}$;

(f) Thickness dependent attenuation efficiencies to X-ray photons with energy of $50 \mathrm{keV}^{[30]}$ 


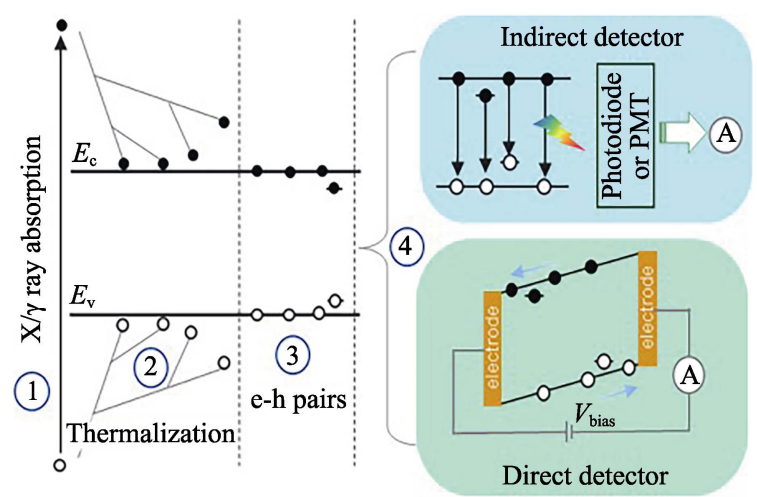

图 $3 \mathrm{X} / \gamma$ 射线与卤化物钲钛矿材料相互作用及直接、间接 射线探测器工作原理示意图(1) (4)为射线与材料相互作用的 过程)

Fig. 3 Schematic illustration of direct and indirect radiation detectors, as well as relaxation kinetics of $\mathrm{X} / \gamma$-ray photons generated carriers in halide perovskite (1)-(4) represent the interaction process between rays and materials)

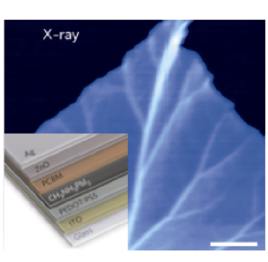
Hybrid PVK film
$(\mathrm{X}-\text {-ray })^{[21]}$ 2015

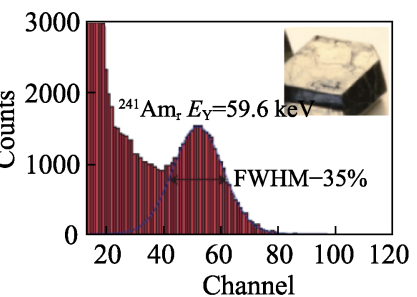

Hybrid PVK crystal $(\gamma \text {-ray spectrometer })^{[20]}$ 2016

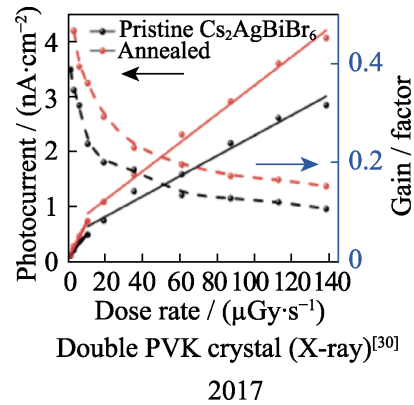

2017

$\mathrm{A}_{2} \mathrm{~B}^{+} \mathrm{B}^{3+} \mathrm{X}_{6}$ 能在一定程度上解决目前铅卤钙钛 矿材料的两个核心问题——稳定性和毒性。Pan 等 ${ }^{[30]}$ 最先将 $\mathrm{Cs}_{2} \mathrm{AgBiBr}_{6}$ 单晶 $(373 \mathrm{~K}$ 氮气中退火 $2 \mathrm{~h})$ 应用 于 $\mathrm{X}$ 射线探测器。 $\mathrm{Cs}_{2} \mathrm{AgBiBr}_{6}$ 含重元素 $\mathrm{Bi}$, 平均 $Z$ 值为 53.1, 高于 $\mathrm{MAPbI}_{3}(48.9) 、 \mathrm{MAPbBr}_{3}(45.1)$ 及 $\alpha-\mathrm{Se}(34)$, 可以更有效地衰减 $\mathrm{X}$ 射线; $\mathrm{Cs}_{2} \mathrm{AgBiBr}_{6}$ 载 流子寿命长 (光致发光寿命 $660 \mathrm{~ns}$, 而 $\mathrm{MAPbBr}_{3}$ 为 $357 \mathrm{~ns}$ ) ${ }^{[14,63]}$, 器件暗电流低(电阻率 $10^{9} \sim 10^{11} \Omega \cdot \mathrm{cm}$, 而 $\mathrm{MAPbBr}_{3}$ 为 $10^{7} \sim 10^{8} \Omega \cdot \mathrm{cm}$ ); 无机单晶可避免有 机离子迁移的问题, 随剂量增加, 光电流下降而增益 因子增大(图 6(a)), 探测器检测限降到 $59.7 \mathrm{nGy}_{\mathrm{air}} \cdot \mathrm{s}^{-1}$ (缺陷密度为 $1.74 \times 10^{9} \mathrm{~cm}^{-3}, \mu \tau$ 为 $6.3 \times 10^{-3} \mathrm{~cm}^{2} \cdot \mathrm{V}^{-1}$ )。 Steele 等 ${ }^{[64]}$ 进一步采用液氮制冷 $(\sim 77 \mathrm{~K})$ 来退激活浅 能级缺陷、降低声子散射。 $\mathrm{Cs}_{2} \mathrm{AgBiBr}_{6}$ 器件灵敏度在 $100 \mathrm{~K}$ 时达到最大(约 $1000 \mu \mathrm{C} \cdot \mathrm{Gy}^{-1} \cdot \mathrm{cm}^{-2}$ )(图 6(b))。

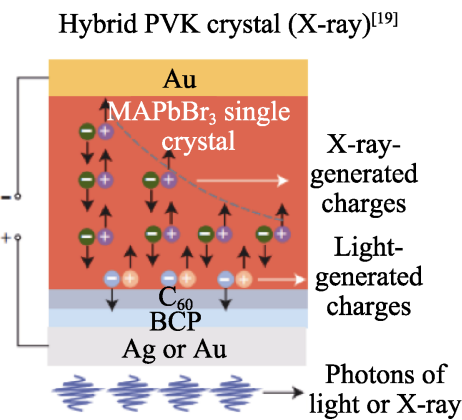

Printable hybrid MPC
(X-ray imaging $)^{[33]}$

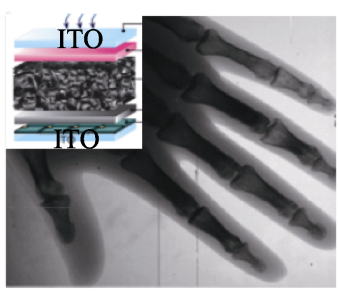

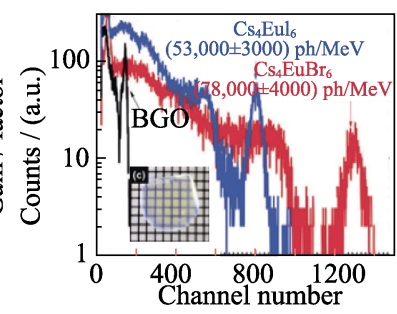

0D inorganic PVK crystal scintillator $(\gamma \text {-ray spectrometer })^{[34]}$ 2018

图 4 卤化物钲钛矿射线探测器发展历程 ${ }^{[19-21,30,32-34]}$

Fig. 4 Development of halide perovskite based radiation detectors ${ }^{[19-21,30,32-34]}$
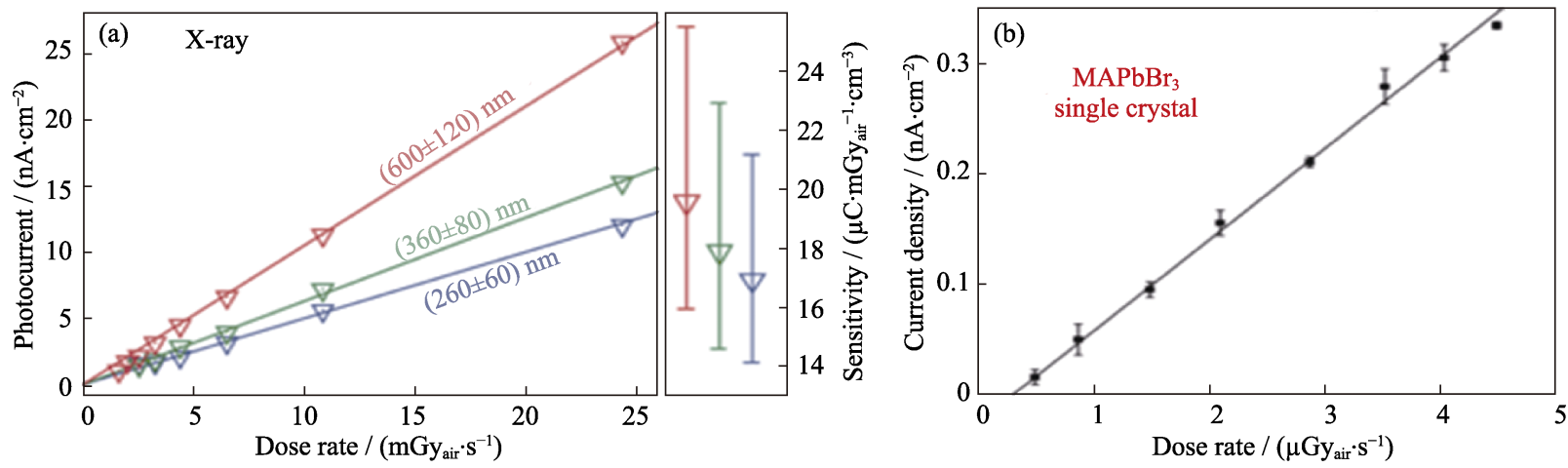

图 5 (a) $\mathrm{MAPbI}_{3}$ 多晶膜( $(260 \pm 60) \mathrm{nm}$ 厚)探测器的 $\mathrm{X}$ 射线 $(37 \mathrm{keV})$ 光电流响应 ${ }^{[21]} ;(\mathrm{b}) \mathrm{MAPbBr}_{3}$ 单晶( $2 \mathrm{~mm}$ 厚) 探测器 $X$ 射线 $(50 \mathrm{keV})$ 的光电流与射线剂量的关系 ${ }^{[19]}$

Fig. 5 (a) Time-resolved photocurrent of $\mathrm{MAPbI}_{3}$ film based detector under $37 \mathrm{keV} \mathrm{X-ray}{ }^{[21]}$; (b) Photocurrent of $\mathrm{MAPbBr}_{3}$ single crystal detector versus dose of $50 \mathrm{keV}$ X-ray ${ }^{[19]}$ 

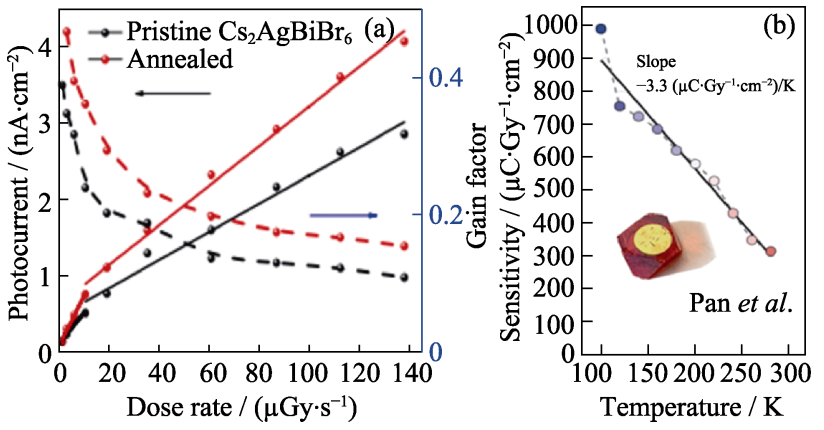

图 6 双钻钛矿 $\mathrm{Cs}_{2} \mathrm{AgBiBr}_{6}$ 单晶 $(2 \mathrm{~mm}$ 厚)的 $\mathrm{X}$ 射线 $(30 \mathrm{keV})$ 响应特性(a)X 射线光电流和增益因子与剂量的关系 ${ }^{[30]}$; (b) 探 测灵敏度对工作温度的依赖性(插图为探测器照片 ${ }^{[64]}$ )

Fig. 6 Response characteristics of $\mathrm{Cs}_{2} \mathrm{AgBiBr}_{6}$ single crystal ( $2 \mathrm{~mm}$ thickness) based X-ray $(30 \mathrm{keV})$ detector. (a) X-ray photocurrent and gain factor as a function of dose rate ${ }^{[30]}$; (b) Temperature dependence of detector sensitivity with inset showing the photograph of single crystal device ${ }^{[64]}$

如前所述，双钙铁矿材料存在两个不同的 B 位金 属离子, 导致双钙钛矿材料存在两种不同的 $\left[\mathrm{BX}_{6}\right]$, 进而从理论计算以及实验角度出发, 双钻钛矿材料 的能级结构以及光电性能更加难以评估, 所以目前 合成的卤化物双钙钛矿种类较少 ${ }^{[65]}$, 而且现已合成 的双钙钛矿材料的带隙都较大 $(1.02 \sim 3.9 \mathrm{eV})^{[63,66-80]}$, 虽然可以通过掺杂等手段来调节带隙, 但由于对双 钙钛矿材料的形成机理以及掺杂机理的研究还不够 深入, 掺杂元素种类的选择及比例的调节都需进一 步研究。如何匹配调节好两个 $\mathrm{B}$ 位金属离子之间的 性能、生长高质量的薄膜或单晶双钙钣矿材料仍是 未来双钙钛矿材料广泛应用面临的主要难题。

\subsection{2 成像应用}

利用高能射线强的穿透性及生物组织/材料对 射线吸收阻止能力的差异, 射线成像是卤化物射线 探测器最重要的应用之一。在成像方面, 卤化物钻 钛矿具有传统 HPGe、CZT 等不具备的独特优势: 1)兼容性, 钙钣矿可通过低温溶液法生长在 $\mathrm{CCD}$ 、 TFT 等成像芯片上 “原位” 集成；2)大尺寸，不受 (HPGe、CZT)尺寸制约，多晶浆料/单晶生长可向上 拓展; 3)柔性兼容。
自从 2015 年 Yakunin 等 ${ }^{[21]}$ 首先展示了树叶、芯 片成像以来, 手掌、胶囊、鱼尾等 $\mathrm{X}$ 光成像相继被 报道, 如图 7(a c ) 。 Kim 等 ${ }^{[33]}$ 合成了与单晶体形貌 及光电性质相近的大晶粒可打印多晶钙钛矿 (MPC), 可印刷在 TFT 芯片上; Wei 等 ${ }^{[81}$ 报道了 $\mathrm{MAPbBr}_{3}$ 单 晶在 $\mathrm{Si}$ 基底上的生长, 在 $8 \mathrm{keV}$ 射线照射下，探测 器的灵敏度达到了 $2.1 \times 10^{4} \mu \mathrm{C} \cdot \mathrm{Gy}^{-1} \cdot \mathrm{cm}^{-2}$, 是传统 $\alpha$-Se 探测器的 1000 倍以上, 可将医学检测的剂量降 至现有剂量的 $1 / 15 \sim 1 / 120 ; \mathrm{Li}$ 等 ${ }^{[82]}$ 使用简单的滴铸 工艺获得了大面积、致密的 $\mathrm{Cs}_{2} \mathrm{AgBiBr}_{6} / \mathrm{PVA}$ 复合 柔性射线探测器, 如图 7(d)所示。

综上，卤化物钻钠矿的高射线吸收系数、高灵 敏及低温溶液法生长等特性与优势, 有利于超薄 (柔性)、高灵敏、高清晰射线相机的开发, 有望大幅 减轻医学影像、安检过程的辐射风险。表 1 总结了 2015 年以来钙钛矿射线探测器的代表性成果。短短 4 年, 探测器的性能就有了明显的提升, 并继续向低 辐射剂量、高灵敏度、稳定、无毒的方向不断发展。

\subsection{3 能谱测量}

能谱是测定原子/核素种类的重要手段, 射线能 谱在材料科学、核物理、高能天体物理及环境放射 性核素监测等领域都有重要的应用。 (a)

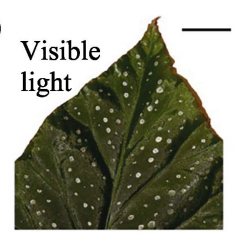

(b)

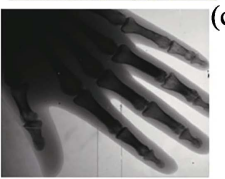

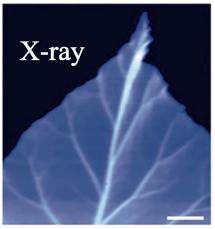

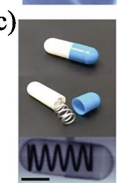

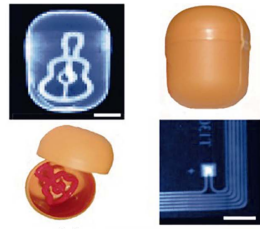

(d)

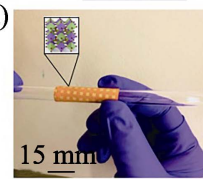

图 7 (a)树叶和电子芯片的 $X$ 射线图像 ${ }^{[21]}$; (b) 手掌 $X$ 射线图 像 ${ }^{[33]}$; (c) 封装金属弹簧和鱼尾鳍光学(上)和 X 射线图像 $(\text { 下 })^{[81]}$; (d) $\mathrm{Cs}_{2} \mathrm{AgBiBr}_{6} / \mathrm{PVA}$ 复合膜柔性探测器 ${ }^{[30,82]}$

Fig. 7 X-ray images of (a) leaf and electronic chip ${ }^{[21]}$; (b) A hand phantom ${ }^{[33]}$; (c) An encapsulated metallic spring and a portion of a fish caudal fin ${ }^{[81]}$; (d) $\mathrm{Cs}_{2} \mathrm{AgBiBr}_{6} / \mathrm{PVA}$ composite film based flexible X-ray detector ${ }^{[30,82]}$

表 1 钙钛矿射线探测器性能比较

Table 1 Comparison of perovskite $X / \gamma$-ray detectors

\begin{tabular}{|c|c|c|c|c|c|c|c|}
\hline Compound & $\begin{array}{c}\text { Dose/ } \\
\left(\mu \mathrm{C} \cdot \mathrm{Gy} \cdot \mathrm{s}^{-1}\right)\end{array}$ & $\begin{array}{c}\text { Sensitivity/ } \\
\left(\mu \mathrm{C} \cdot \mathrm{Gy}^{-1} \cdot \mathrm{cm}^{-2}\right)\end{array}$ & $\begin{array}{c}\text { Energy/ } \\
\text { keV }\end{array}$ & $\begin{array}{c}\mu \tau / \\
\left(\mathrm{cm}^{2} \cdot \mathrm{V}^{-1}\right)\end{array}$ & $\begin{array}{c}\text { Mobility/ } \\
\left(\mathrm{cm}^{2} \cdot \mathrm{V}^{-1} \cdot \mathrm{s}^{-1}\right)\end{array}$ & $\begin{array}{l}\text { Resistivity/ } \\
(\Omega \cdot \mathrm{cm})\end{array}$ & Ref. \\
\hline $\mathrm{MAPbI}_{3}$ (film) & - & $2.5 \times 10^{4}$ & 8 & $2 \times 10^{-7}$ & - & - & {$[21]$} \\
\hline $\mathrm{MAPbBr}_{3}$ (single crystal) & 0.5 & 80 & 50 & $1.2 \times 10^{-2}$ & - & - & [19] \\
\hline $\mathrm{MAPbBr}_{3} / \mathrm{Si}$ & - & $2.1 \times 10^{4}$ & 8 & - & 201 & - & [81] \\
\hline $\mathrm{Cs}_{2} \mathrm{AgBiBr}_{6}$ (single crystal) & 0.0597 & - & & $6.3 \times 10^{-3}$ & 11.81 & - & [30] \\
\hline $\mathrm{Cs}_{2} \mathrm{AgBiBr}_{6}$ (single crystal) & 0.0597 & 105 & 50 & - & - & $3.6 \times 10^{12}$ & [64] \\
\hline
\end{tabular}


能谱测量要求探测器有高灵敏度及能量分辨 率。2016 年首次报道了 $\mathrm{MAPbI}_{3}(\mu \tau=(1.0 \sim 1.8) \times$ $\left.10^{-2} \mathrm{~cm}^{2} \cdot \mathrm{V}^{-1}\right) 、 \mathrm{MAPbBr}_{3} 、 \mathrm{MAPbCl}_{3} 、 \mathrm{FAPbI}_{3} 、 \mathrm{FAPbBr}_{3}$ 单晶的 $\gamma$ 射线能谱 ${ }^{[20]}$, 可测得 ${ }^{241} \mathrm{Am}(59.6 \mathrm{keV})$ 的能 谱, 其相对分辨率达到 35\%(图 8(a,b))。随后, $\mathrm{He}$ 等 ${ }^{[83]}$ 采用逆温度梯度结晶法生长了高质量 $\mathrm{MAPbI}_{3}$ 单晶 ( $4 \mathrm{~mm} \times 3 \mathrm{~mm} \times 1.52 \mathrm{~mm}, \mu \tau=\sim 0.8 \times 10^{-3} \mathrm{~cm}^{2} \cdot \mathrm{V}^{-1}$ ), 采 用功函数差别较大的 $\mathrm{Ga}(4.3 \mathrm{eV}) 、 \mathrm{Au}(\sim 5.1 \mathrm{eV})$ 非对 称电极, 在 $\mathrm{MAPbI}_{3}$ 两端形成 $\mathrm{Ga} / \mathrm{MAPbI}_{3} / \mathrm{Au}$ 肖特 基接触。在反向偏压(耗尽)下测试, 探测器对 ${ }^{57} \mathrm{Co}$ $(122 \mathrm{keV})$ 的能量分辨率达到 6.8\%(图 8(c))。此外, Huang 等 ${ }^{\left[{ }^{[4]}\right.}$ 利用卤素掺杂材料中的载流子，在 $\mathrm{MAPbBr}_{3}$ 中掺入 $6 \%$ 的 $\mathrm{Cl}$ 可显著降低缺陷浓度、提 升载流子浓度, 提升器件对 ${ }^{137} \mathrm{Cs}$ 的能谱响应, 接近 商用的 $\mathrm{NaI}(\mathrm{Tl})$ 探测器(图 8(d))。

2018 年, $\mathrm{He}$ 等 ${ }^{[85]}$ 用布里奇曼法生长了大尺寸 $\mathrm{CsPbBr}_{3}$ 单晶(杂质总浓度在 $10^{-5}$ 以下)。同样使用非对 称 $\mathrm{Ga} / \mathrm{CsPbBr} 3 / \mathrm{Au}$ 肖特基接触, 器件对 ${ }^{57} \mathrm{Cs}(122 \mathrm{keV})$ (图 9(a)) 的相对能量分辨率达 3.9\%。器件对 32.3 $662 \mathrm{keV}$ 的 $\mathrm{X} / \gamma$ 射线光子都有较好的识别性能(图 9(b))。此外, Nazarenko 等 ${ }^{86]}$ 还展示了便携式钲钛矿
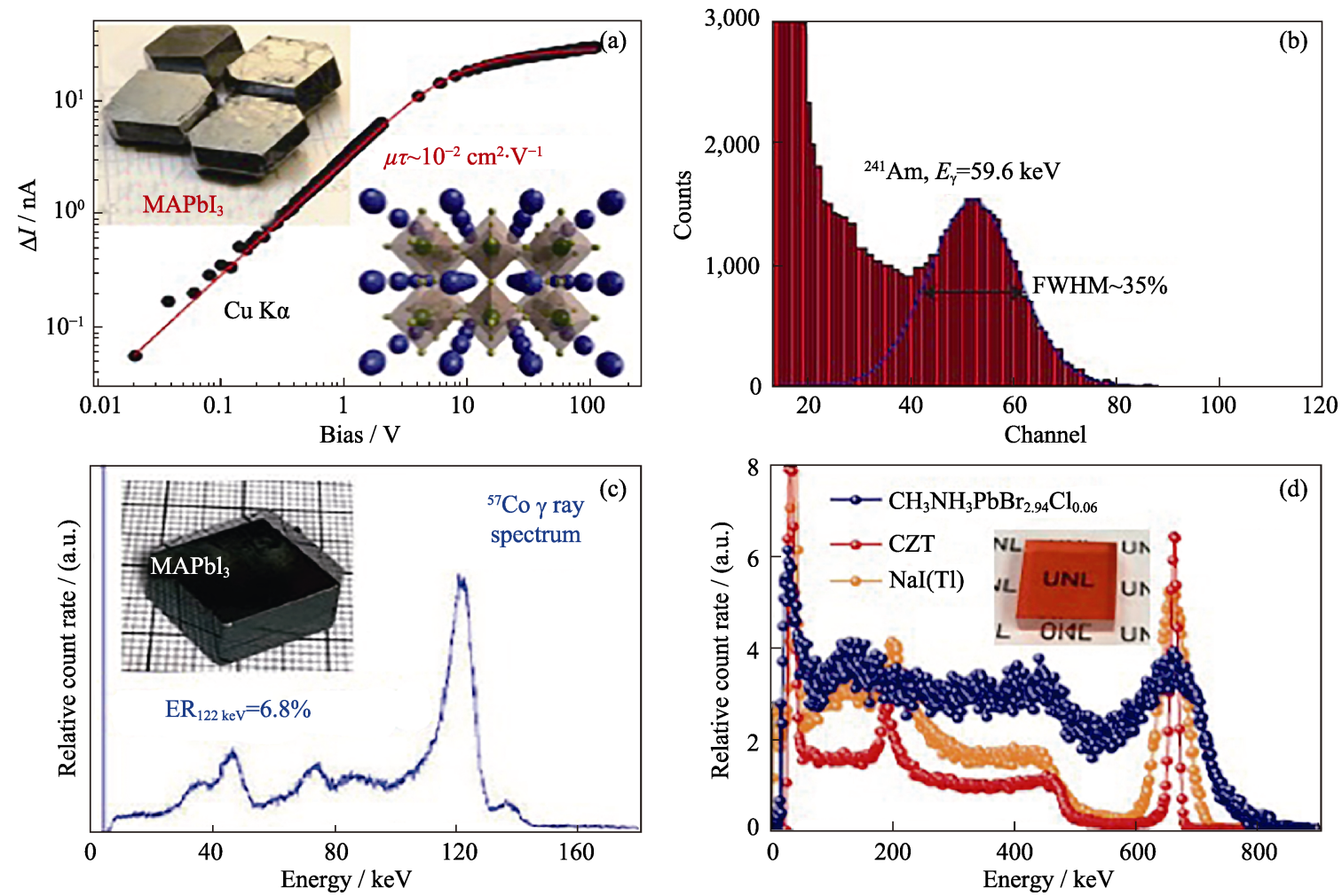

图 8 有机-无机杂化钲钣矿单晶探测器的 $\gamma$ 射线能谱 $(a) \mathrm{MAPbI}_{3}$ 单晶探测器 $\mu \tau$ 测量 ${ }^{[20]}$; (b) 单晶探测器对 ${ }^{241} \mathrm{Am}$ 的能谱响应 ${ }^{[20]}$; (c) $\mathrm{MAPbI}_{3}$ 单晶探测器对 ${ }^{57} \mathrm{Co}$ 的能谱响应 ${ }^{[83]}$; (d) MAPbBr $2{ }_{244} \mathrm{Cl}_{0.06}$ 探测器、CZT、 $\mathrm{NaI}(\mathrm{Tl})$ 探测器对 ${ }^{137} \mathrm{Cs}$ 的能谱响应 ${ }^{[84]}$

Fig. $8 \quad \gamma$-ray spectroscopy of single crystal based organic-inorganic hybrid perovskite detector. (a) The bias dependence of the photocurrent generated by $\mathrm{Cu} \mathrm{K} \alpha \mathrm{X}$-ray in a $\mathrm{FAPbI}_{3}$ single crystal ${ }^{[20]}$; (b) Energy spectroscopy curve of single crystal detector for ${ }^{241} \mathrm{Am}^{[20]}$; (c) $\mathrm{MAPbI}_{3}$ single crystal photo and the spectral response of ${ }^{57} \mathrm{Co}^{[83]}$; (d) The spectral response of $\mathrm{MAPcBr}_{2.94} \mathrm{Cl}_{0.06}$ detector, $\mathrm{CZT}$, NaI (Tl) detector to ${ }^{137} \mathrm{Cs}^{[84]}$ 

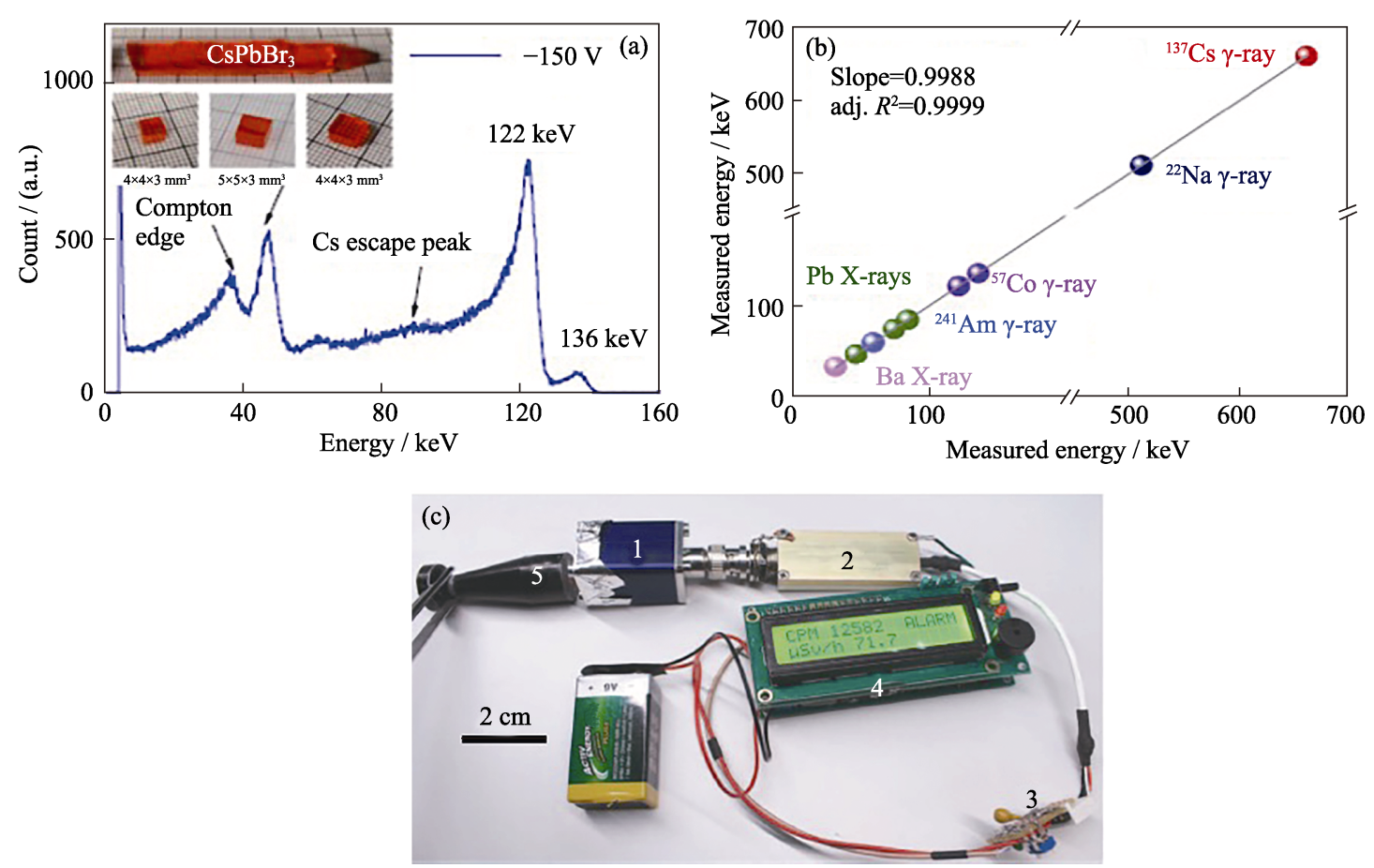

图 9 (a)布里奇曼法生长的全无机 $\mathrm{CsPbBr}_{3}$ 单晶及对 ${ }^{137} \mathrm{Cs}$ 的能谱响应曲线 ${ }^{[85]}$; (b)单晶探测器线性响应范围 ${ }^{[85]}$; (c)便携式( $\mathrm{FA} / \mathrm{Cs}) \mathrm{Pb}(\mathrm{I} / \mathrm{Br})_{3} \gamma$ 射线能谱仪 (1: 单晶探头, 2: 前置放大器, 3: 放大器, 4: 显示器, 5: 射线源, 6: $9 \mathrm{~V}$ 干电池) ${ }^{[86]}$

Fig. 9 (a) $\gamma$-ray energy spectrum of $\mathrm{CsPbBr}_{3}$ single crystal grown by Bridgman method toward ${ }^{137} \mathrm{Cs}^{[85]}$;

(b) Linear response range of the detector ${ }^{[85]}$; (c) Portable $(\mathrm{FA} / \mathrm{Cs}) \mathrm{Pb}(\mathrm{I} / \mathrm{Br})_{3} \gamma$-ray spectrometer ${ }^{[86]}$

1: single crystal probe, 2: preamplifier, 3: amplifier, 4: display, 5: ray source, 6: battery

表 2 卤化物射线探测器能谱检测性能(ITC: 逆温度梯度结晶法)

Table 2 Halide $\gamma$-ray detector energy spectroscopy detection summary (ITC: Inverse temperature gradient crystallization)

\begin{tabular}{|c|c|c|c|c|c|c|c|c|}
\hline Compound & $\begin{array}{l}\text { Growth } \\
\text { method }\end{array}$ & $\begin{array}{c}\text { Thickness/ } \\
\text { mm }\end{array}$ & Electrode & $\mu \tau /\left(\mathrm{cm}^{2} \cdot \mathrm{V}^{-1}\right)$ & $\begin{array}{l}\text { Resistivity/ } \\
(\Omega \cdot \mathrm{cm})\end{array}$ & $\begin{array}{l}\text { Electric field/ } \\
\left(\mathrm{V} \cdot \mathrm{mm}^{-1}\right)\end{array}$ & $\begin{array}{l}\text { Energy } \\
\text { resolution }\end{array}$ & Ref. \\
\hline $\mathrm{MAPbI}_{3}$ & ITC & 3 & - & $(1.0-1.8) \times 10^{-2}$ & - & 3 & $35 \%$ for ${ }^{241} \mathrm{Am}$ & [20] \\
\hline $\mathrm{MAPb} \mathrm{Br}_{2.94} \mathrm{Cl}_{0.06}$ & ITC & $2-2.4$ & $\mathrm{Cr} / \mathrm{C}_{60} / \mathrm{BCP} / \mathrm{PVK} / \mathrm{Cr}$ & $1.8 \times 10^{-2}$ & $3.6 \times 10^{9}$ & 1.8 & $24 \%$ for ${ }^{137} \mathrm{Cs}$ & [84] \\
\hline $\mathrm{MAPbI}_{3}$ & ITC & 1.52 & $\mathrm{Ga} / \mathrm{PVK} / \mathrm{Au}$ & $8 \times 10^{-4}$ & $10^{8}-10^{9}$ & $33-46$ & $6.8 \%$ for ${ }^{57} \mathrm{Co}$ & [83] \\
\hline $\mathrm{CsPbBr}_{3}$ & Bridgman & $0.9-3$ & $\mathrm{Ga} / \mathrm{PVK} / \mathrm{Au}$ & $1.34 \times 10^{-3}$ & - & 167 & $\begin{array}{l}3.9 \% \text { for }{ }^{57} \mathrm{Co} \\
3.8 \% \text { for }{ }^{137} \mathrm{Cs}\end{array}$ & [85] \\
\hline
\end{tabular}

高性能的闪炼体单晶通常由高温熔融法生长, 生长工艺复杂、温度高、无法实现闪炼体探测器 的“原位”集成。2016 年, Birowosuto 等 ${ }^{[88]}$ 首先报 道了有机一无机杂化卤化物钙钛矿 (单晶) 闪胨体性 能。由于钙钛矿(单晶)材料的激子束缚能较低, 室 温下荧光热淬灭效应严重, 导致射线荧光效率较 低(10 $10^{3}$ photon $\left./ \mathrm{MeV}\right)$, 只能在低温 $(10 \mathrm{~K})$ 下观 察到明显的可见光辐射 $\left(\mathrm{MAPbBr}_{3}\right.$ 可见光产率 $1.5 \times 10^{5}$ photon $/ \mathrm{MeV} ; \mathrm{MAPbI}_{3}$ 在 $750 \mathrm{~nm}$ 的苂光产 率可达到 $1.5 \times 10^{5}$ photon $\left./ \mathrm{MeV}\right)$ 。2018 年, 新加坡 国立大学、西北工业大学、福州大学等多个课题组 ${ }^{[87]}$ 采用激子束缚能更大的 $\mathrm{CsPbX}_{3}$ 来克服此问题 (图 10(a)), 并充分利用量子点的量子效应(将射线 激发的电子-空穴对限域在粒径小于波尔半径的量 子点(图 10(b)), 极大地提升了射线激发电子-空穴
的辐射复合几率, 成功地展示了室温下工作的高 苂光效率闪炼体。通过调节卤素组分, 获得覆盖可 见光波段(380 700 $\mathrm{nm}$ ) 准连续可调的高效闪炼体 (图 10(c f f))。此外, $X$ 射线光子通过卤化铅钻钛矿 纳米晶体材料中的直接带隙发射转换成多种低能 量可见光子(图 10(a)), 探测器展现出较低的检测 限 (13 nGy $\left.\cdot \mathrm{s}^{-1}\right)$ 和较短的苂光驰豫时间 (44.6 ns) (图 10(g, h))。这种柔性基板可实现快速 $\mathrm{X}$ 射线多 色显示(图 10(e, f)), 是常规块体闪炼体无法实现 的。这些工作表明, 通过选择高激子束缚能的钙钛 矿材料, 进一步利用量子限域效应，可高效操控射 线激发电子-空穴对的辐射复合几率，拓展卤化物 钙铁矿在闪炼体探测器中的应用, 为开发新型高 灵敏、多彩、低成本、大面积、柔性兼容射线成像 提供了新思路。 

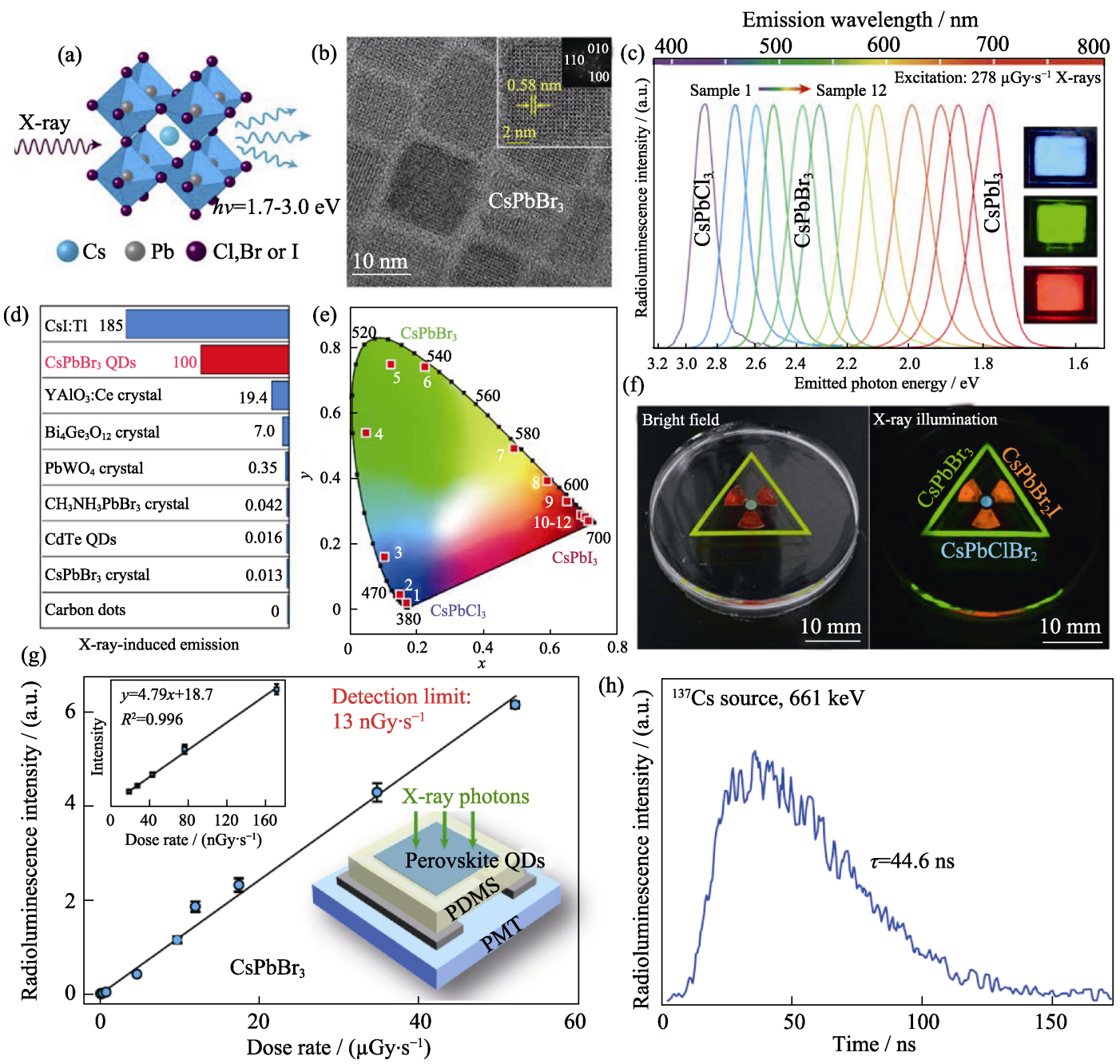

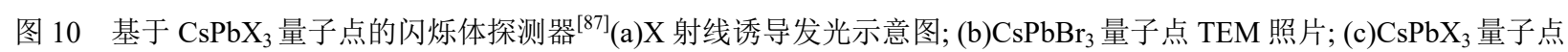

在 $50 \mathrm{keV}$ (剂量 $278 \mu \mathrm{Gy} \cdot \mathrm{s}^{-1}$ )X 射线辐照下的可调谐发光光谱; (d) $10 \mathrm{kV}$ 射线辐照下闪炼体材料的 X 射线光学灵敏度;

(e)不同组分 $\mathrm{CsPbX}_{3}$ 量子点闪㷧体发光在 $\mathrm{CIE}$ 色度图中的坐标; (f)三色 $\mathrm{CsPbX}_{3}$ 闪伢体在可见光(左)及 $\mathrm{X}$ 射线(右)

激发下的荧光; $(\mathrm{g}) \mathrm{CsPbr}_{3}$ 闪炼体基射线探测剂量曲线; (h) $\mathrm{CsPbr}_{3}$ 闪炼体在 ${ }^{137} \mathrm{Cs}$ 源激发下的辐射发光衰减曲线

Fig. $10 \mathrm{CsPbX}_{3}$ quantum dot based halide perovskite scintillation detector ${ }^{[87]}$. (a) Schematic representation of X-ray-induced luminescence; (b) Transmission electron microscopy image of the as-aynthesized $\mathrm{CsPBr}_{3}$ nanocrystals; (c) Tunable luminescence

spectra of $\mathrm{CsPbX}_{3}$ quantum dots under X-ray irradiation at $50 \mathrm{keV}$ (dose rate of $278 \mu \mathrm{Gy} \cdot \mathrm{s}^{-1}$ ); (d) Comparison of the optical sensitivity of various scintillator materials in response to exposure to X-rays produced at a voltage of $10 \mathrm{kV}$; (e) CIE chromaticity coordinates of the X-ray-induced visible emissions of $12 \mathrm{CsPbX}_{3}$ samples; (f) Multicolour X-ray scintillation from 3 types of perovskite nanocrystal scintillator; ( $\mathrm{g}$ ) $\mathrm{CsPbBr}_{3}$-based scintillator as a function of dose rate; (h) Measured radioluminescence decay of the $\mathrm{CsPbr}_{3}$-based scintillator under excitation with a ${ }^{137} \mathrm{Cs}$ source

\section{3 钙钛矿射线探测应用关键问题分析}

材料是射线探测器的核心, 高性能的射线探测 器依赖材料的综合性能: 射线吸收系数、载流子迁 移率、载流子寿命、(热、化学)稳定性、带隙、缺 陷浓度等。因此, 开发综合光电性能优异的卤化物 钙钛矿材料是获得高性能射线探测器的关键。

物相决定了晶体的性能, 由于影响晶体物相的 因素较多(原料的比例、溶液或反溶剂的种类及比 例、反应温度、气体氛围、压力等), 因此优化晶体 生长条件是获得高质量的大尺寸晶体的重要手段;
材料的稳定性和毒性是目前卤化物钙钠矿材料面临 的两大核心问题: 1)热稳定性和化学稳定性差。这主 要是因为有机阳离子在高温、潮湿条件下极易和水、 氧气等发生反应, 导致卤化物钻钛矿材料的面心立 方结构发生改变, 从而影响器件的性能。虽然加热 干燥等手段可除去溶剂中多余的水分, 良好的封装 技术可有效隔绝空气，但制备成本会大幅增长，同 时也不能保证器件的长期稳定。因此从材料本身性 质出发, 通过掺杂无机离子来提升稳定性更为有 效。然而目前缺乏清晰的掺杂机理来指导如何选择 和控制掺杂元素种类和比例。深入理解掺杂机理、 
优化掺杂方法是主要的研究思路。2)材料的毒性。 铅元素独特的电子结构决定了铅基卤化物钙铁矿材 料的优良性能, 但铅元素无法自然降解, 会对环境 造成严重污染, 不符合材料的绿色环保要求。用与 铅元素电子组成类似的金属元素来取代铅, 是一个 可行的方法。然而目前对非铅基钙钛矿材料自身结 构以及非铅元素的电子状态的研究都不够深入, 所 以目前难以合成兼顾无毒(无铅)与高光电性能的钲 钛矿材料。深入研究非铅基钙钛矿材料的结构特性 是解决这一问题的关键。为了进一步推进卤化物钙 铁矿材料在新兴领域的应用, 今后仍需继续利用材 料基因组计算及实验手段, 优化界面(调控层与层 之间的能级匹配, 抑制钙钣矿分解 ${ }^{89]}$ ), 开发对应的 表面缺陷钝化、欧姆/肖特基接触的新方法与手段, 进一步提升钙钛矿探测器的检测剂量、灵敏度、信 噪比、响应时间和稳定性等性能。

\section{4 结束语}

卤化物钙钛矿的高射线吸收系数、高迁移率、 高载流子寿命及材料特有的可低温溶液法制备特性, 为射线探测器(强度探测、高清成像、能谱)的低成 本、高效、原位集成提供了可能。在过去短短四年 内，卤化物钙钛矿射线探测器在探测极限、灵敏度、 能量分辨率等方面都取得了长足的进步，部分性能 指标优于现阶段商用的半导体射线探测器。此外, 卤化物钙钛矿 (尤其是双钙钛矿)组分的多样性也为 今后设计综合性能更优异的射线探测器材料提供了 新的机遇, 从而推动射线探测器在医学成像、安防 安检和环境放射性检测等领域的深入应用。

\section{参考文献:}

[1] KASAP S, FREY J B, BELEV G, et al. Amorphous and polycrystalline photoconductors for direct conversion flat panel X-ray image sensors. Sensors (Basel), 2011, 11(5): 5112-5157.

[2] SAKDINAWAT A, ATTWOOD D. Nanoscale X-ray imaging. $\mathrm{Na}$ ture Photonics, 2010, 4(12): 840-848.

[3] SZELES C. CdZnTe and CdTe materials for X-ray and gamma ray radiation detector applications. Physica Status Solidi B-basic Research, 2004, 241(3): 783-790.

[4] KASAP S O, KABIR M Z, ROWLANDS J A, et al. Dependence of the detective quantum efficiency of photoconductive X-ray image detectors on charge transport parameters and exposure: application to $\alpha$-Se. Applied Physics Letters, 2002, 81 (18): 3482-3484.

[5] ZHANG P, YANG J, XWEI S H. Manipulation of cation combinations and configurations of halide double perovskites for solar cell absorbers. Journal of Materials Chemistry A, 2018, 6(4): $1809-1815$.

[6] XIAOLING W, ZUYONG F, NAN W, et al. Progress on novel perovskite solar cells. Materials China, 2016, 35(12): 960-965.
[7] CHENG Z, YLIN J. Layered organic-inorganic hybrid perovskites: structure, optical properties, film preparation, patterning and templating engineering. CrystEngComm, 2010, 12(10): 2646-2662.

[8] BHALLA A S, GUO R, YROY R. The perovskite structure: a review of its role in ceramic science and technology. Materials Research Innovations, 2000, 4(1): 3-26.

[9] ZHANG B, YAN J, WANG J, et al. Effect of the modulating of organic content on optical properties of single-crystal perovskite. Optical Materials, 2016, 62: 273-278.

[10] WANG Z, SHI Z J, LI T T, et al. Stability of perovskite solar cells: a prospective on the substitution of the acation and xanion. Angewandte Chemie-International Edition, 2017, 56(5): 1190-1212.

[11] JENA A K, KULKARNI AMIYASAKA T. Halide perovskite photovoltaics: background, status, and future prospects. Chemical Reviews, 2019, 119(5): 3036-3103.

[12] YIN W J, YANG J H, KANG J, et al. Halide perovskite materials for solar cells: a theoretical review. J. Mater. Chem. A, 2015, 3(17): 8926-8942.

[13] ZHAO X G, YANG J H, FU Y H, et al. Design of lead-free inorganic halide perovskites for solar cells via cation-transmutation. Journal of The American Chemical Society, 2017, 139(7): 2630-2638.

[14] SHI D, ADINOLFI V, COMIN R, et al. Low trap-state density and long carrier diffusion in organolead trihalide perovskite single crystals. Science, 2015, 347(6221): 519-522.

[15] HAO F, STOUMPOS C C, CHANG R P H, et al. Anomalous band gap behavior in mixed $\mathrm{Sn}$ and $\mathrm{Pb}$ perovskites enables broadening of absorption spectrum in solar cells. Journal of The American Chemical Society, 2014, 136(22): 8094-8099.

[16] HE J L, VASENKO A S, LONG R, et al. Halide composition controls electron-hole recombination in cesium-lead halide perovskite quantum dots: a time domain ab initio study. Journal of Physical Chemistry Letters, 2018, 9(8): 1872-1879.

[17] KOVALENKO M V, PROTESESCU LBODNARCHUK M I. Properties and potential optoelectronic applications of lead halide perovskite nanocrystals. Science, 2017, 358(6364): 745-750.

[18] WEI H, THUANG J S. Halide lead perovskites for ionizing radiation detection. Nat. Commun. 2019, 10(1): 1066.

[19] WEI H T, FANG Y J, MULLIGAN P, et al. Sensitive X-ray detectors made of methylammonium lead tribromide perovskite single crystals. Nature Photonics, 2016, 10(5): 333-339.

[20] YAKUNIN S, DIRIN D N, SHYNKARENKO Y, et al. Detection of gamma photons using solution-grown single crystals of hybrid lead halide perovskites. Nature Photonics, 2016, 10(9): 585-589.

[21] YAKUNIN S, SYTNYK M, KRIEGNER D, et al. Detection of $\mathrm{X}$-ray photons by solution-processed organic-inorganic perovskites Nature Photonics, 2015, 9(7): 444-449.

[22] LIAN Z P, YAN Q F, GAO T T, et al. Perovskite $\mathrm{CH}_{3} \mathrm{NH}_{3} \mathrm{PbI}_{3}(\mathrm{Cl})$ single crystals: rapid solution growth, unparalleled crystalline quality, and low trap density toward $10^{8} \mathrm{~cm}^{-3}$. Journal of The American Chemical Society, 2016, 138(30): 9409-9412.

[23] DONG Q F, FANG Y J, SHAO Y C, et al. Electron-hole diffusion lengths $>175 \mu \mathrm{m}$ in solution-grown $\mathrm{CH}_{3} \mathrm{NH}_{3} \mathrm{PbI}_{3}$ single crystals. Science, 2015, 347(6225): 967-970.

[24] DENG Y H, PENG E, SHAO Y C, et al. Scalable fabrication of efficient organolead trihalide perovskite solar cells with doctorbladed active layers. Energy \& Environmental Science, 2015, 8(5): 1544-1550.

[25] LONG RPREZHDO O V. Dopants control electron-hole recombination at perovskite- $\mathrm{TiO}_{2}$ interfaces: $A b$ initio time-domain study. ACS Nano, 2015, 9(11): 11143-11155.

[26] ZHUMEKENOV A A, SAIDAMINOV M I, HAQUE M A, et al. Formamidinium lead halide perovskite crystals with unprecedented 
long carrier dynamics and diffusion length. ACS Energy Letters, 2016, 1(1): 32-37.

[27] YETTAPU G R, TALUKDAR D, SARKAR S, et al. Terahertz conductivity within colloidal $\mathrm{CsPbBr}_{3}$ perovskite nanocrystals: remarkably high carrier mobilities and large diffusion lengths. Nano Letters, 2016, 16: 4838-4848.

[28] MiYATA A, Mitioglu A, PLOCHOCKA P, et al. Direct measurement of the exciton binding energy and effective masses for charge carriers in organic-inorganic tri-halide perovskites. Nature Physics, 2015, 11(7): 582-587.

[29] D'INNOCENZO V, GRANCINI G, ALCOCER M J P, et al. Excitons versus free charges in organo-lead tri-halide perovskites. Nat. Commun. 2014, 5: 3586.

[30] PAN W C, WU H D, LUO J J, et al. $\mathrm{Cs}_{2} \mathrm{AgBiBr}_{6}$ single-crystal Xray detectors with a low detection limit. Nature Photonics, 2017, 11(11): 726-732.

[31] HU X, ZHANG X, LIANG L, et al. High-performance flexible broadband photodetector based on organolead halide perovskite. Advanced Functional Materials, 2014, 24(46): 7373-7380.

[32] HEO J H, SHIN D H, PARK J K, et al. High-performance nextgeneration perovskite nanocrystal scintillator for nondestructive X-ray imaging. Advanced Materials, 2018, 30(40): e1801743.

[33] KIM Y C, KIM K H, SON D Y, et al. Printable organometallic perovskite enables large-area, low-dose X-ray imaging. Nature, 2017, 550(7674): 87-91.

[34] WU Y T, HAN D, CHAKOUMAKOS B C, et al. Zero-dimensional $\mathrm{Cs}_{4} \mathrm{EuX}_{6}(\mathrm{X}=\mathrm{Br}, \mathrm{I})$ all-inorganic perovskite single crystals for gamma-ray spectroscopy. Journal of Materials Chemistry C, 2018, 6(25): 6647-6655.

[35] ZHOU H W, NIE Z H, YIN J, et al. Antisolvent diffusion-induced growth, equilibrium behaviours in aqueous solution and optical properties of $\mathrm{CH}_{3} \mathrm{NH}_{3} \mathrm{PbI}_{3}$ single crystals for photovoltaic applications. RSC Advances, 2015, 5(104): 85344-85349.

[36] HUANG J S, SHAO Y, CDONG Q F. Organometal trihalide perovskite single crystals: a next wave of materials for $25 \%$ efficiency photovoltaics and applications beyond? Physical Chemistry Letters, 2015, 6(16): 3218-3227.

[37] SU J, CHEN D, PLIN C T. Growth of large $\mathrm{CH}_{3} \mathrm{NH}_{3} \mathrm{PbX}$ (X=I, Br) single crystals in solution. Journal of Crystal Growth, 2015, 422: 75-79.

[38] RONG Y, TANG Z, ZHAO Y, et al. Solvent engineering towards controlled grain growth in perovskite planar heterojunction solar cells. Nanoscale, 2015, 7(24): 10595-10599.

[39] EISEN Y, SHOR A, MARDOR I. CdTe and CdZnTe X-ray and $\gamma$-ray detectors for imaging systems. IEEE Transactions on $\mathrm{Nu}$ clear Science, 2004, 51(3): 1191-1198.

[40] DVORYANKIN V F, DVORYANKINA G G, KUDRYASHOV A A, et al. X-ray sensitivity of $\mathrm{Cd}_{0.9} \mathrm{Zn}_{0.1} \mathrm{Te}$ detectors. Technical Physics, 2010, 55(2): 306-308.

[41] SHEARER D, RBOPAIAH M. Dose rate limitations of integrating survey meters for diagnostic X-ray surveys. Health Physics, 2000, 79(2 Suppl): S20-21.

[42] CLAIRAND I, BORDY J M, CARINOU E, et al. Use of active personal dosemeters in interventional radiology and cardiology: tests in laboratory conditions and recommendations-oramed project. Radiation Measurements, 2011, 46(11): 1252-1257.

[43] CONINGS B, DRIJKONINGEN J, GAUQUELIN N, et al. Intrinsic thermal instability of methylammonium lead trihalide perovskite. Advanced Energy Materials, 2015, 5: 1500477.

[44] YIN W J, CHEN H Y, SHI T T, et al. Origin of high electronic quality in structurally disordered $\mathrm{CH}_{3} \mathrm{NH}_{3} \mathrm{PbI}_{3}$ and the passivation effect of $\mathrm{Cl}$ and $\mathrm{O}$ at grain boundaries. Advanced Electronic Mate- rials, 2015, 1: 1500044 .

[45] STOUMPOS C C, MALLIAKAS C D, PETERS J A, et al. Crystal growth of the perovskite semiconductor $\mathrm{CsPbBr}_{3}$ : a new material for high-energy radiation detection. Crystal Growth \& Design, 2013, 13(7): 2722-2727.

[46] YUAN Y, BHUANG J S. Ion migration in organometal trihalide perovskite and its impact on photovoltaic efficiency and stability. Accounts of Chemical Research, 2016, 49(2): 286-293.

[47] LEE J W, KIM D H, KIM H S, et al. Formamidinium and cesium hybridization for photo- and moisture-stable perovskite solar cell. Advanced Energy Materials, 2015, 5: 1501310.

[48] YI C Y, LUO J S, MELONI S, et al. Entropic stabilization of mixed a-cation $\mathrm{ABX}_{3}$ metal halide perovskites for high performance perovskite solar cells. Energy \& Environmental Science, 2016, 9(2): 656-662.

[49] CHEN C Y, LIN H Y, CHIANG K M, et al. All-vacuum-deposited stoichiometrically balanced inorganic cesium lead halide perovskite solar cells with stabilized efficiency exceeding $11 \%$. Advanced Materials, 2017, 29 (12): 1605290.

[50] WU Y, HUANG Y, CAO F, et al. Capping $\mathrm{CsPbBr}_{3}$ with $\mathrm{ZnO}$ to improve performance and stability of perovskite memristors. Nano Research, 2017, 10(5): 1584-1594.

[51] LI X, YU D, WANG Y, et al. Constructing fast carrier tracks into flexible perovskite photodetectors to greatly improve responsivity. ACS Nano, 2017, 11(2): 2015-2023.

[52] XUE J, GU Y, SHAN Q, et al. Constructing mie-scattering porous interface-fused perovskite films to synergistically boost light harvesting and carrier transport. Angewandte Chemie International Edition, 2017, 56: 5232-5236.

[53] HUANG C Y, ZOU C, MAO C, et al. $\mathrm{CsPbBr}_{3}$ perovskite quantum dot vertical cavity lasers with low threshold and high stability. ACS Photonics, 2017, 4(9): 2281-2289.

[54] HUO C, LIU X, SONG X, et al. Field-effect transistors based on van-der-Waals-grown and dry-transferred all-inorganic perovskite ultrathin platelets. Journal of Physical Chemistry Letters, 2017, 8: 4785-4792.

[55] LIU D, LIN Q, ZANG Z, et al. Flexible all-inorganic perovskite $\mathrm{CsPbBr}_{3}$ nonvolatile memory device. ACS Applied Materials \& Interfaces, 2017, 9: 6171-6176.

[56] PANIGRAHI S, JANA S, CALMEIRO T, et al. Imaging the anomalous charge distribution inside $\mathrm{CsPbBr}_{3}$ perovskite quantum dots sensitized solar cells. ACS Nano, 2017, 11: 10214-10221.

[57] ZHANG L, YANG X, JIANG Q, et al. Ultra-bright and highly efficient inorganic based perovskite light-emitting diodes. Nature Communication, 2017, 8: 15640 .

[58] YANG B, ZHANG F, CHEN J, et al. Ultrasensitive and fast allinorganic perovskite-based photodetector via fast carrier diffusion. Advanced Materials, 2017, 29(40): 1703758.

[59] PROTESESCU L, YAKUNIN S, BODNARCHUK M I, et al. Nanocrystals of cesium lead halide perovskites $\left(\mathrm{CsPbX}_{3}, \mathrm{X}=\mathrm{Cl}, \mathrm{Br}\right.$, and I): novel optoelectronic materials showing bright emission with wide color gamut. Nano Letters, 2015, 15(6): 3692-3696.

[60] ZHANG Z Q, FENG X, LU X, et al. Polymer-passivated inorganic cesium lead mixed-halide perovskites for stable and efficient solar cells with high open-circuit voltage over 1.3 V. Advanced Materials, 2018, 30(9): 1705393.

[61] YANG T, ZHENG Y, DU Z, et al. Superior photodetectors based on all-inorganic perovskite $\mathrm{CsPb}_{3}$ nanorods with ultrafast re-sponse and high stability. ACS Nano, 2018, 12(2): 1611-1617.

[62] LOU-WEN Z, SHAO-LI S, LU-YING L, et al. Application and development of cesium lead halide perovskite based planar heterojunction leds. Journal of Inorganic Materials, 2019, 34: 37-48. 
[63] SLAVNEY A H, HU T, LINDENBERG A M, et al. A bismuthhalide double perovskite with long carrier recombination lifetime for photovoltaic applications. Journal of The American Chemical Society, 2016, 138(7): 2138-2141.

[64] STEELE J A, PAN W C, MARTIN C, et al. Photophysical pathways in highly sensitive $\mathrm{Cs}_{2} \mathrm{AgBiBr}_{6}$ double-perovskite single-crystal X-ray detectors. Advanced Materials, 2018, 30(46): e1804450.

[65] CHU L, AHMAD W, LIU W, et al. Lead-free halide double perovskite materials: a new superstar toward green and stable optoelectronic applications. Nano-Micro Letters., 2019, 11: 16.

[66] LEE B, STOUMPOS C C, ZHOU N J, et al. Air-stable molecular semiconducting lodosalts for solar cell applications: $\mathrm{Cs}_{2} \mathrm{SnI}_{6}$ as a hole conductor. Journal of the American Chemical Society, 2014, 136(43): 15379-15385.

[67] SAPAROV B, SUN J P, MENG W W, et al. Thin-film deposition and characterization of a Sn-deficient perovskite derivative $\mathrm{Cs}_{2} \mathrm{SNi}_{6}$. Chemistry of Materials, 2016, 28(7): 2315-2322.

[68] MAUGHAN A E, GANOSE A M, BORDELON M M, et al. Defect tolerance to intolerance in the vacancy-ordered double perovskite semiconductors $\mathrm{Cs}_{2} \mathrm{SNi}_{6}$ and $\mathrm{Cs}_{2} \mathrm{TeI}_{6}$. Journal of The American Chemical Society, 2016, 138(27): 8453-8464.

[69] KALtZOGLOU A, ANTONIADOU M, KONTOS A G, et al. Optical-vibrational properties of the $\mathrm{Cs}_{2} \mathrm{SnX}_{6}(\mathrm{X}=\mathrm{Cl}, \mathrm{Br}, \mathrm{I})$ defect perovskites and hole-transport efficiency in dye-sensitized solar cells. Journal of Physical Chemistry C, 2016, 120(22): $11777-$ 11785 .

[70] LEE B, KRENSELEWSKI A, BAIK S I, et al. Solution processing of air-stable molecular semiconducting iodosalts, $\mathrm{Cs}_{2} \mathrm{SnI}_{6}-x \mathrm{Br}_{x}$, for potential solar cell applications. Sustainable Energy \& Fuels, 2017, 1(4): 710-724.

[71] JU M G, CHEN M, ZHOU Y Y, et al. Earth-abundant nontoxic titanium (IV)-based vacancy-ordered double perovskite halides with tunable 1.0 to $1.8 \mathrm{eV}$ bandgaps for photovoltaic applications. ACS Energy Letters, 2018, 3(2): 297-304.

[72] ZHANG J, YU C H, WANG L L, et al. Energy barrier at the N719dye/ $\mathrm{CsSNi}_{3}$ interface for photogenerated holes in dye-sensitized solar cells. Scientific Reports, 2014, 4: 6954.

[73] CLARK R J, HTRUMBLE W R. Resonance raman-spectra of some mixed-valence halogeno-compounds of antimony and lead. Journal of The Chemical Society-Dalton Transactions, 1976, 12(12): 11451149.

[74] MAUGHAN A E, GANOSE A M, CANDiA A M, et al. Anharmonicity and octahedral tilting in hybrid vacancy-ordered double perovskites. Chemistry of Materials, 2018, 30(2): 472-483.

[75] MAUGHAN A E, GANOSE A M, ALMAKER M A, et al. Tolerance factor and cooperative tilting effects in vacancy-ordered dou- ble perovskite halides. Chemistry of Materials, 2018, 30(11): 3909-3919.

[76] SAKAI N, HAGHIGHIRAD A A, FILIP M R, et al. Solutionprocessed cesium hexabromopalladate (IV), $\mathrm{Cs}_{2} \mathrm{PdBr}_{6}$, for optoelectronic applications. Journal of The American Chemical Society, 2017, 139(17): 6030-6033.

[77] EVANS H A, FABINI D H, ANDREWS J L, et al. Hydrogen bonding controls the structural evolution in perovskite-related hybrid platinum (IV) iodides. Inorganic Chemistry, 2018, 57(16): 10375- 10382.

[78] Kaltzoglou A, ANTONiadou M, PERGANTi D, et al. Mixed-halide $\mathrm{Cs}_{2} \mathrm{SnI}_{3} \mathrm{Br}_{3}$ perovskite as low resistance hole-transporting material in dye-sensitized solar cells. Electrochimica Acta, 2015, 184: 466-474.

[79] ZHANG H N, GAO Z Y, LIANG X R. X-ray detector based on all-inorganic lead-free $\mathrm{Cs}_{2} \mathrm{AgBiBr}_{6}$ perovskite single crystal. IEEE Transactions on Electron Devices, 2019, 66(5): 2224-2229.

[80] DAY P. Spectra and constitution of antimony (III), antimony (V) hexahalide salts and related compounds. Molecules into Materials, 2007, 2: 120-124.

[81] WEI W, ZHANG Y, XU Q, et al. Monolithic integration of hybrid perovskite single crystals with heterogenous substrate for highly sensitive X-ray imaging. Nature Photonics, 2017, 11(5): 315-321.

[82] LI H R, SHAN X, NEU J N, et al. Lead-free halide double perovskite-polymer composites for flexible X-ray imaging. Journal of Materials Chemistry C, 2018, 6(44): 11961-11967.

[83] HE Y H, KE W J, ALEXANDER G C B, et al. Resolving the energy of gamma-ray photons with mapbi(3) single crystals. ACS Photonics, 2018, 5(10): 4132-4138.

[84] WEI H T, DESANTIS D, WEI W, et al. Dopant compensation in alloyed $\mathrm{CH}_{3} \mathrm{NH}_{3} \mathrm{PbBr}_{3-x} \mathrm{Cl}_{x}$ perovskite single crystals for gammaray spectroscopy. Nature Materials, 2017, 16(8): 826-833.

[85] HE Y H, MATEI L, JUNG H J, et al. High spectral resolution of gamma-rays at room temperature by perovskite $\mathrm{CsPbBr}_{3}$ single crystals. Nat. Commun., 2018, 9(1): 1609.

[86] NAZARENKO O, YAKUNIN S, MORAD V, et al. Single crystals of caesium formamidinium lead halide perovskites: solution growth and gamma dosimetry. NPG Asia Materials, 2017, 9: e373.

[87] CHEN Q S, WU J, OU X Y, et al. All-inorganic perovskite nanocrystal scintillators. Nature, 2018, 561(7721): 88-93.

[88] BIROWOSUTO M D, CORTECCHIA D, DROZDOWSKI W, et al. X-ray scintillation in lead halide perovskite crystals. Sci. Rep., 2016, 6: 37254 .

[89] SHAN X, WANG S, MENG G, et al. Interface engineering of electron transport layer/light absorption layer of perovskite solar cells. Progress in Chemistry, 2019, 31(5): 714-722. 